\title{
Propiedades Psicométricas de la Versión en Español de la Prueba Procrastination Assessment Scale-Students (PASS)
}

\author{
Psychometric Properties of the Spanish Version of the Test Procrastination \\ Assessment Scale-Students (PASS)
}

Angélica Garzón Umerenkova ${ }^{1}$ y Javier Gil Flores ${ }^{2}$

\begin{abstract}
Resumen
Se analizan las características psicométricas de la versión española de la prueba Procrastination Assessment Scale-Students (PASS), adaptada para el alumnado universitario colombiano. Se exploran la validez de contenido y constructo, mediante el modelo Rasch; la validez factorial, identificando los factores subyacentes a las razones para procrastinar, y la validez discriminante, mediante el análisis de la relación con las variables gestión del tiempo y calificaciones académicas. También se analizan la confiabilidad, la longitud de la escala y el comportamiento diferencial de los ítems (DIF). La muestra estuvo constituida por 494 universitarios de nueve carreras. Los resultados indican un buen ajuste al modelo Rasch, valores adecuados de confiabilidad, ítems sin funcionamiento diferencial, la posibilidad de reducción de la escala Likert a cuatro posibilidades de respuesta y el hallazgo de cinco factores que dan cuenta de la razones para procastinar. Se obtuvieron correlaciones negativas y significativas entre la procrastinación y la gestión del tiempo y las calificaciones.
\end{abstract}

Palabras clave: procrastinación académica, modelo Rasch, rendimiento académico

\begin{abstract}
This paper analyze the psychometric properties of the Spanish version of the test Procrastination Assessment Scale-Students (PASS) applied to college students in Colombia. The content and construct validity through Rasch model, the factorial validity through factor analysis of reasons to procrastinate and the discriminant validity over the analysis of the relationship with time management and grades was researched. Also, the test reliability, lenght of Likert scale and differential item functioning (DIF) was analyzed. The sample was 494 college students from nine different programs. The results indicate that the data fits the model adequately, has good values of reliability, the DIF was not found in any items, the Likert scale could be reduced from five to four categories, and five factors explained the reasons to procrastinate. Negative and significant correlations between procrastination and time management and grades was obtained.
\end{abstract}

Keywords: academic procrastination, Rasch model, academic performance

\footnotetext{
${ }^{1}$ Facultad de Psicología, Universidad El Bosque. Av. Cra 9 No. 131 A-02, Bogotá, Colombia. Tel.: 00573118879075. Correo: agarzonu@gmail.com

${ }^{2}$ Facultad Ciencias de la Educación. C/ Pirotecnia, s/n, 41013-Sevilla, España. Tel.: 0034955 20635. Correo: jflores@us.es
} 


\section{Introducción}

Procrastinar es la tendencia a gastar el tiempo, demorar y aplazar de forma intencionada algo que debe ser hecho (Tuckman, 2003). El procrastinador tiende a elegir los beneficios a corto plazo sobre las ganancias a largo plazo, lo que indica una pobre autorregulación (Wilson y Nguyen, 2012) por lo que la procrastinación se entiende como un fallo en la autorregulación del individuo (Steel, 2007).

La procrastinación aparece con una alta frecuencia en diferentes culturas, particularmente en el ámbito académico. En la universidad se calcula que entre el $70 \%$ y el $95 \%$ de los estudiantes procrastinan (Klassen \& Kuzucu, 2009; Ferrari, O'Callaghan, \& Newbegin, 2005; Steel, 2007). Se trata, además, de una conducta particularmente estable, que se mantiene desde el inicio hasta el final del semestre académico (Rice, Richardson, \& Clark, 2012).

Se han encontrado relaciones entre una alta procrastinación y una tendencia a una baja autoeficacia percibida por los estudiantes sobre la autorregulación y las tareas académicas. De tal manera que los alumnos que presentan mayor confianza en sus habilidades académicas (niveles elevados de autoeficacia) tienden a procrastinar menos. La autoeficacia está relacionada a su vez con el uso de herramientas de planificación e inicio de las tareas de manera más oportuna (Wolters, 2003). Hay evidencia de que los procrastinadores frecuentes subestiman los esfuerzos destinados a las tareas actuales sobre el éxito final en la obtención de una meta (Dewitt, \& Schouwenburg, 2002).

Por otro lado, dada su naturaleza autorregulatoria, la procrastinación se ve afectada por la motivación hacia la tarea. Las tareas que son percibidas como difíciles, poco atractivas, ambiguas y que requieren más esfuerzo que otras, presentan mayor probabilidad de ser objeto de procrastinación (Ferrari, Mason, \& Hammer, 2006).

Niveles elevados de procrastinación se han relacionado también con altos niveles de ansiedad, estrés general y estrés relacionado con enfermedad (Klassen \& Kuzucu, 2009). Burka y Yuen (2008) alegan que en la base de toda procrastinación estaría presente una respuesta aprendida de miedo que genera una conducta de evitación, con independencia de los niveles de estrés impuestos por la tarea académica.

En términos generales, los reportes en la literatura acerca de las razones para procrastinar pueden agruparse en cinco categorías: atracción por la tarea, amor al trabajo, incertidumbres sobre la tarea, miedo al fracaso/fallo en la tarea (Zarick \& Stonebraker, 2009) y perfeccionismo, ya sea para presentar un resultado satisfactorio (autoperfeccionismo) o para impresionar a otros.

En las revisiones meta-analíticas de Steel (2007) y Rozental y Carlbring (2014), en el conjunto de las investigaciones reportadas en el área se confirma una consistente relación negativa entre la procrastinación y el rendimiento académico. Sin embargo, esta relación se ve afectada no solo por el diseño de la investigación, sino, además, por el instrumento de medida empleado para evaluar procrastinación y por una serie de variables moderadoras (por ejemplo, edad, tema del curso, exigencias de la asignatura, características del alumno: como motivación, capacidad de automonitoreo, valor de la tarea, etc.). El estudio de estas relaciones constituye, en consecuencia, un fructífero campo de investigación actual.

Por otro lado, a pesar del hecho que cada investigación puede utilizar un indicador de rendimiento académico diferente, consistentemente los procrastinadores muestran correlaciones negativas con el promedio general acumulado, el promedio de un curso específico, los resultados en un examen final o las calificaciones de las tareas, según sea la medida de rendimiento usada en las investigaciones. Por el contrario, los estudiantes con baja tendencia a procrastinar tienen más probabilidades de finalizar a tiempo sus estudios universitarios de primer año, en comparación con los de alta tendencia a procrastinar (Bruinsma \& Jansen, 2009). Esta relación inversa entre la procrastinación y el rendimiento académico se presenta incluso por encima de variables tradicionalmente asociadas a la ejecución académica. Wang y Englander (2010) hallaron en estudiantes de primer año universitario matriculados en un curso de estadística que la procrastinación tuvo un impacto más significativo 
en la ejecución que la asistencia a clase y la nota de ingreso a la universidad.

Es necesario considerar, de cara al manejo preventivo de los factores que afectan el rendimiento académico del alumnado, que la procrastinación puede disminuir con una intervención efectiva. Diversas evidencias apuntan a que las habilidades de mejora en el manejo del tiempo y la disminución de la procrastinación son alcanzables mediante un entrenamiento sistemático (Britton \& Tesser 1991; Gortner \& Zulauf 2000; Kitsantas, Winsler, \& Huie, 2008; Macan, Shahani, Dipboye, \& Phillips, 1990; Pehlivan, 2013; Robbins, Oh, Le, \& Button, 2009). Como indican Rozental y Carlbring (2014) para la intervención en contextos educativos hay disponibles técnicas cognitivo conductuales, provenientes de la práctica clínica y de los programas de manejo del tiempo, probadas con éxito en el alumnado para disminuir la procrastinación. Por ejemplo, el establecimiento de rutinas y calendarios; el manejo de las creencias irracionales que alimentan la procrastinación; la exposición paulatina a los eventos que evita el alumno al procrastinar; el establecimiento de metas fraccionadas en objetivos diarios para aumentar la autodisciplina y la motivación; el aumento de la autoeficacia percibida de los estudiantes, entre otras. Las intervenciones tradicionalmente son presenciales $\mathrm{y}$ en grupos relativamente pequeños, pero actualmente se está estudiando el uso de técnicas virtuales o no presenciales para la disminución de la procrastinación (Glick \& Orsillo, 2015; Rozental, Forsström, Nilsson, Rizzo, \& Carlbring, 2014). Es posible que la investigación en el área se incline cada vez más hacia el desarrollo de intervenciones efectivas remotas que lleguen a más estudiantes en menor tiempo y costo.

Finalmente, autores como Moore (2008) argumentan que la procrastinación reportada por el estudiante mediante cuestionarios puede ser un fuerte predictor de la ejecución académica. Sin embargo hay que considerar que existen diversos instrumentos de autorreporte para la evaluación de la procrastinación, académica y no académica, tales como la Escala de Procrastinación (Tuckman, 1991), la Escala de Procrastinación Pura (Steel, 2010), la Escala General de
Procrastinación (Lay, 1986) o la Escala de Decisión de Procrastinación (Mann, 1982 citado por Díaz-Morales, Ferrari, Díaz, \& Argumedo, 2006), entre otros. Sin embrago, a pesar de su potencial como predictores del rendimiento académico, la mayoría de estos instrumentos no han sido adaptados al español, ni en poblaciones estudiantiles latinoamericanas. Una excepción en pruebas de manejo del tiempo es la adaptación al portugués del inventario de perspectiva temporal en estudiantes universitarios (Nunes, 2012).

En un contexto en el que crece el interés por tener disponibles cada vez más pruebas con demostradas propiedades psicométricas (ver, por ejemplo, Carvalho \& Novo, 2014; Dufey \& Fernandez, 2012) el objeto de este estudio fue caracterizar psicométricamente la prueba PASS (Procrastination Assessment Scale-Students) creada por Solomon y Rothblum (1984) en una muestra de alumnado universitario colombiano. Como señalan sus autoras, este cuestionario, a diferencia de otros que también miden procrastinación académica, incorpora otras posibles variables o razones para procrastinar, por lo cual la conceptualización de la procrastinación que subyace al PASS va más allá de un deficiente manejo del tiempo, situación que proporciona una visión más completa de las diferentes razones que pueden estar generando en los estudiantes las demoras en la realización de las tareas.

Este estudio pretende obtener un instrumento con unos indicadores psicométricos definidos de: validez de constructo de acuerdo a los parámetros del modelo Rasch (dimensionalidad y ajuste al modelo), índices de confiabilidad, análisis del funcionamiento diferencial de los ítems, estimación del parámetro b, comprobación de la longitud de la escala Likert, validez factorial de las razones para procrastinar y evidencia de validez discriminante entre la frecuencia de procrastinación con la gestión del tiempo y las calificaciones académicas, para que eventualmente la prueba pueda ser usada para la evaluación de la procrastinación en universitarios hispanohablantes de nuevo ingreso. 


\section{Método}

\section{Muestra}

La población objeto de este estudio fue el alumnado colombiano de primeros semestres universitarios. Se realizó un muestreo por conveniencia pero se tuvieron en cuenta, por una parte, el número de participantes necesarios para responder al objetivo del estudio y, por otra, la necesidad de dotar a la muestra de un nivel de heterogeneidad, considerando dos instituciones universitarias (Universidad El Bosque y Uniempresarial), varias titulaciones, y las dos jornadas de estudios (diurna y nocturna). La muestra estuvo constituida por 494 estudiantes universitarios. Una vez aplicada la encuesta sociodemográfica se obtuvieron los resultados que describen las principales características del alumnado participante. Los 494 alumnos tenían edades comprendidas entre los 16 y 46 años; 127 hombres y 367 mujeres de primer año universitario. Corresponden al primer semestre 442 estudiantes y al segundo 49 estudiantes. Por otro lado, 347 pertenecían a la jornada diurna y 147 a la jornada nocturna. De acuerdo a las carreras la muestra estuvo constituida de la siguiente forma: Psicología (291 estudiantes), Educación Bilingüe (16), Pedagogía (31), Ingeniería de Sistemas (39), Ingeniería Electrónica (19), Administración de Empresas (21), Finanzas y Comercio Exterior (53), Negocios Internacionales (10) y Marketing (11). Los estudiantes participaron de manera voluntaria en el estudio, a los cuales se les hizo firmar un consentimiento previo a su participación.

\section{Procedimiento}

La aplicación de las pruebas fue colectiva en las aulas de informática a través de una plataforma Web construida para la investigación en el año 2012. Los participantes contaron con tiempo ilimitado y la posibilidad de un periodo de descanso entre encuestas. La mayoría de los estudiantes tardó menos de una hora y durante la aplicación al menos un investigador siempre estuvo presente para resolver las inquietudes de los participantes.

\section{Instrumentos}

La prueba PASS (Solomon y Rothblum, 1984) consta de 44 ítems y se divide en dos secciones. La primera parte de la prueba tiene 18 ítems que se dirigen a determinar la frecuencia de la procrastinación y el grado en el cual ésta culmina en ansiedad. Divide la procrastinación por actividades académicas (por ejemplo, preparar un examen o un trabajo) y, a su vez, indaga sobre cuánta molestia o tensión le genera al estudiante el aplazar cada una de estas actividades. Las opciones de respuesta se presentan en una escala Likert con valores de 1 a 5 con descriptores de 1 (nunca), 2 (casi nunca), 3 (a veces), 4 (casi siempre) y 5 (siempre). La segunda sección del PASS abarca del ítem 19 al 44. Estos ítems indagan sobre las razones cognitivo-conductuales para procrastinar. Las opciones de respuesta se presentan en un escala Likert con valores de 1 a 5 donde 1 significa "no refleja mis motivos en absoluto", 3 significa "los refleja hasta cierto punto" y 5 significa "los refleja perfectamente". El estudio inicial de Solomon y Rothblum (1984) encontró dos claros factores que agrupaban las razones para procrastinar: (1) razones de ansiedad, perfeccionismo y baja autoconfianza, que explicaba el 49,4\% de la varianza y (2) razones de aversión a la tarea y pereza, que explicó el $18 \%$ de la varianza. Las autoras informaron de un tercer factor que agrupaba las demás razones para procrastinar, pero que fue descartado por el escaso porcentaje de varianza explicada. Ferrari (1989) encontró que los coeficientes test-retest del PASS fueron significativos para el índice de prevalencia de la procrastinación $(\mathrm{r}=.74, p=.001)$ y el índice total de razones para procrastinar $(\mathrm{r}=.56, p=.001)$.

Para su aplicación en Colombia, un traductor profesional realizó la traducción del PASS, esta primera versión del inglés al español fue revisada por tres psicólogos colombianos en busca de expresiones confusas o imprecisiones. Posteriormente, se aplicó la versión final a cinco estudiantes universitarios colombianos a quienes se les realizó una entrevista semiestructurada al final de la aplicación de la prueba en busca de términos confusos o ambiguos desde el punto de vista cultural. Las preguntas que constituyeron la entrevista semiestructurada fueron las siguientes: "Entendiste todo el contenido del cuestionario?/ 
Existe algún término o palabra que no hayas comprendido del todo?/ Por cuál palabra reemplazarías el término que te genera confusión?/ Quieres comentar o añadir algo más?". Como resultado del procedimiento descrito se realizaron pequeños ajustes a algunas expresiones concretas, por ejemplo: "conseguir tarjeta de identificación" se cambió por "sacar el carnet".

Para valorar la validez discriminante del PASS se obtuvieron medidas de gestión del tiempo y calificaciones de los estudiantes al final del periodo académico (promedio ponderado).

Para evaluar gestión del tiempo se utilizó la traducción al castellano del TMB (García-Ros \& Pérez-González, 2012). Esta prueba fue creada por Macan et al. (1990), consta de 34 ítems; las cuatro subescalas que integran el TMB (Macan et al., 1990; Macan, 1994) son: fijar metas y prioridades, herramientas para la gestión del tiempo, preferencia por la desorganización y percepción del control del tiempo; que dieron cuenta del $72 \%$ de la varianza y la consistencia interna global de la prueba fue de 0,68. En la adaptación del TMB a lengua castellana con población universitaria española (García Ros \& Pérez-González, 2012) se encontró que la estructura factorial coincide básicamente con la del instrumento original.

\section{Análisis estadísticos}

Se realizó un análisis mediante el modelo Rasch para calibración de los ítems de la prueba de procrastinación mediante el paquete estadístico Winsteps versión 3.72.3. Se hizo un análisis de las propiedades psicométricas: dimensionalidad, ajuste al modelo, parámetro b, confiabilidad, funcionamiento de la escala y análisis DIF.

El modelo Rasch se asienta básicamente en dos principios: la unidimensionalidad y la independencia local. La unidimensionalidad permite la estimación de la existencia de un factor principal del instrumento y la independencia local indica que las respuestas de las personas a cualquier pregunta son independientes de sus respuestas a otra pregunta. La escala en Loguitos (en inglés, logits scale) es una representación de la habilidad del individuo, quien responde a los ítems de la prueba en diferentes magnitudes de dificultad (Bond y Fox, 2012). Los estadísticos de ajuste son el criterio de medias cuadráticas (MNSQ) para identificar el peso o valor de la información (infit) y la sensibilidad a los extremos (outfit). Los valores del MNSQ pueden ir de cero hasta el infinito con un valor esperado de 1 .

Como se indicó en la sección de instrumento, la prueba PASS tiene dos secciones claramente diferenciadas (la primera parte mide frecuencia de la procrastinación y la segunda las razones para procrastinar) por lo que se procedió a realizar los análisis de estos dos atributos (dos partes de la prueba) de manera separada mediante el modelo Rasch.

Por otra parte, para establecer la estructura de las razones para procrastinar, se realizó un análisis de los factores componentes mediante un análisis factorial exploratorio para la segunda parte de la prueba. Como señala Messick (1989) la validación de una prueba psicológica abarca todas las cuestiones experimentales, estadísticas, teóricas y filosóficas por medio de las cuales se evalúan las hipótesis y teorías científicas. En dicho procedimiento de validación, el análisis factorial es la técnica estadística por excelencia usada para la validación de constructo.

Finalmente, para obtener evidencia de la validez discriminante del PASS, se correlacionaron las puntuaciones de la primera parte del PASS (frecuencia) con el rendimiento académico y las dimensiones de la prueba de gestión del tiempo (TMB), esperando que la relación fuera inversa con ambas variables (rendimiento y habilidades de gestión del tiempo). Los procedimientos de análisis factorial y correlacional se realizaron mediante el programa estadístico SPSS versión 21.

\section{Resultados}

\section{Dimensionalidad}

Mediante el análisis de componentes principales de residuos se puede llegar a la conclusión de que la prueba sólo mide una dimensión cuando la varianza explicada por la medida es $\geq 40 \%$ (Linacre, 2006); moderada cuando es $\geq 30 \%$ y mínima aceptable cuando es $\geq 20 \%$. En las Tablas 1 y 2 se muestran los resultados del análisis del supuesto de 
unidimensionalidad de cada parte de la prueba, para el primer y segundo atributo, respectivamente.

Tabla 1. Varianza de residuos estandarizados del primer atributo (frecuencia de procrastinación)

\begin{tabular}{lcc}
\hline & Empírica & Modelada \\
\hline Total de varianza bruta $=$ & $100 \%$ & $100 \%$ \\
Varianza bruta explicada por las medidas $=$ & $39.8 \%$ & $39.9 \%$ \\
Varianza bruta explicada por las personas $=$ & $11.2 \%$ & $11.2 \%$ \\
Varianza bruta explicada por las preguntas $=$ & $28.6 \%$ & $28.6 \%$ \\
Varianza bruta sin explicar (total) $=$ & $60.2 \%$ & $60.1 \%$ \\
Varianza bruta sin explicar en 1er contraste $=$ & $13.3 \%$ & $22.1 \%$ \\
Varianza bruta sin explicar en $2^{\circ}$ contraste $=$ & $8.3 \%$ & $13.7 \%$ \\
Varianza bruta sin explicar en 3er contraste $=$ & $6.7 \%$ & $11.2 \%$ \\
Varianza bruta sin explicar en $4^{\circ}$ contraste $=$ & $5.4 \%$ & $8.9 \%$ \\
Varianza bruta sin explicar en $5^{\circ}$ contraste $=$ & $4.7 \%$ & $7.8 \%$ \\
\hline
\end{tabular}

Tabla 2. Varianza de residuos estandarizados del segundo atributo (razones para procrastinar)

\begin{tabular}{lcc}
\hline \multicolumn{1}{c}{ Varianza } & Empírica & Modelada \\
\hline Total de varianza bruta $=$ & $100 \%$ & $100 \%$ \\
Varianza bruta explicada por las medidas $=$ & $37.3 \%$ & $38.3 \%$ \\
Varianza bruta explicada por las personas $=$ & $9.9 \%$ & $10.2 \%$ \\
Varianza bruta explicada por las preguntas $=$ & $27.4 \%$ & $28.2 \%$ \\
Varianza bruta sin explicar (total) $=$ & $62.7 \%$ & $61.7 \%$ \\
Varianza bruta sin explicar en 1er contraste $=$ & $6.6 \%$ & $10.5 \%$ \\
Varianza bruta sin explicar en $2^{\circ}$ contraste $=$ & $5.7 \%$ & $9 \%$ \\
Varianza bruta sin explicar en 3er contraste $=$ & $4.1 \%$ & $6.5 \%$ \\
Varianza bruta sin explicar en $4^{\circ}$ contraste $=$ & $3.6 \%$ & $5.7 \%$ \\
Varianza bruta sin explicar en $5^{\circ}$ contraste $=$ & $13.3 \%$ & $5.3 \%$ \\
\hline
\end{tabular}

La Tabla 1 presenta dos columnas con los valores empíricos y modelados. Para el primer atributo (frecuencia de procrastinación) la varianza explicada por las medidas modeladas es del $39.8 \%$, la varianza explicada por las personas es del $11.2 \%$, la varianza explicada por los ítems es del $28.6 \%$ y la varianza no explicada es del $60.2 \%$. Lo anterior, permite identificar que a pesar de tener un porcentaje tan alto en la varianza no explicada, los tres factores explican más del $50 \%$ de las variaciones de las puntuaciones. Para el segundo atributo (razones para procrastinar), con resultados similares, se encontró que la varianza explicada por las medidas es del $37.3 \%$, la varianza explicada por las personas es del $9.9 \%$, la varianza explicada por los ítems es del $27.4 \%$ y la varianza no explicada es del $62.7 \%$ (ver Tabla 2).

Adicionalmente, se asume como evidencia para la unidimensionalidad de la medida la razón de 3 a 1 para la varianza explicada por la medida sobre la varianza no explicada por el primer contraste (Linacre, 2012). Razón que, como puede observarse en la Tablas 1 y 2 , se cumple para los dos atributos analizados.

\section{Ajuste del modelo}

Se calcula mediante el establecimiento de una expectativa probabilística acorde a la dificultad de cada ítem y a la habilidad de cada persona. El modelo supone que las personas que tienen mayor habilidad responderán correctamente a todos los elementos fáciles, y cuando las personas tienen un nivel de habilidad bajo se espera que no conteste aún a los reactivos más fáciles (Bond \& Fox, 2012). Para este estudio indican ajuste al modelo para ítems y personas valores del infit y outfit entre .5 y 1.5 (Bond \& Fox, 2012). Los ítems fuera del rango de ajuste del MNSQ (media cuadrática) se consideran sobreestimados (la pregunta es muy predecible) o infraestimados (la pregunta es errática).

Para el primer atributo (ver Tabla 3), al contrastar el porcentaje de varianza explicada con los índices de infit y outfit se puede concluir que la bondad de ajuste del modelo de Rasch es buena. Para el segundo atributo (Tabla 4), igualmente, los índices de infit y outfit se encuentran dentro de los rangos permitidos (.5 y 1.5$)$.

\section{Confiabilidad}

Mediante el análisis Rasch se calcula el índice de confiabilidad para los ítems de la prueba y de las personas que son interpretados como un Alfa de Cronbach, esperando valores superiores .80 . Los valores de confiabilidad encontrados para el primer atributo fueron de .99 (ítems) y .86 (sujetos); para el segundo atributo fueron de .89 (ítems) y .91 (sujetos). Otro indicador de confiabilidad es la medida de separación que señala el número de niveles en unidades de error estándar, en el que la muestra de ítems y de personas pueden agruparse. El valor esperado es $\geq 1.5$ (Linacre, 2012). El error de medición de los 
Tabla 3. Estimaciones del infit y outfit del primer atributo (frecuencia de procrastinación) ítems 1 a 18

\begin{tabular}{cccccc}
\hline Ítem & $\begin{array}{c}\text { Puntaje } \\
\text { total }\end{array}$ & Medida & Model S.E. & $\begin{array}{c}\text { INFIT } \\
\text { MNSQ }\end{array}$ & $\begin{array}{c}\text { OUTFIT } \\
\text { MNSQ }\end{array}$ \\
\hline 10 & 1132 & .66 & .05 & 1.18 & 1.24 \\
16 & 1180 & .55 & .05 & .77 & .79 \\
4 & 1196 & .51 & .05 & .96 & 1 \\
11 & 1244 & .41 & .05 & 1.03 & 1.02 \\
13 & 1258 & .38 & .05 & 1.03 & 1.05 \\
17 & 1266 & .36 & .05 & .75 & .76 \\
1 & 1270 & .35 & .05 & .79 & .86 \\
7 & 1273 & .34 & .05 & .83 & .9 \\
14 & 1291 & .3 & .05 & .91 & .93 \\
5 & 1444 & -.02 & .05 & .92 & .93 \\
8 & 1465 & -.06 & .05 & .79 & .79 \\
2 & 1502 & -.13 & .05 & .78 & .78 \\
12 & 1614 & -.37 & .05 & 1.35 & 1.32 \\
18 & 1633 & -.41 & .05 & 1.23 & 1.2 \\
15 & 1661 & -.47 & .05 & 1.13 & 1.1 \\
6 & 1786 & -.74 & .05 & 1.17 & 1.08 \\
9 & 1816 & -.81 & .05 & 1.2 & 1.12 \\
3 & 1830 & -.85 & .05 & 1.32 & 1.24 \\
\hline Media & 1436.7 & 0 & .05 & 1.01 & 1.01 \\
Dt. & 228.1 & .48 & 0 & .2 & .17 \\
\hline
\end{tabular}

Nota: De acuerdo al modelo, para cada una de las preguntas se consideran ajustados al modelo los valores MNSQ (infit y outfit) comprendidos entre 0.5 y 1.5 .

18 ítems del primer atributo es de .05 (ver Tabla 3) y el error correspondiente a los 26 ítems del segundo atributo está entre .05 y .06 (ver Tabla 4).

\section{Análisis del funcionamiento diferencial de los ítems (DIF)}

Para evaluar la invarianza de la medida a través de los subgrupos se estimó el DIF (Funcionamiento Diferencial de los Ítems). Un contraste significativo de DIF es aquel que presenta una diferencia de .5 logitos con $\mathrm{p} \leq .05$ cuando se estima el estadístico de MantelHaenszel para todas las comparaciones (Bond \& Fox, 2012). Al realizar el análisis de DIF por semestres, género y jornada, no se encontró evidencia de funcionamiento diferencial en los ítems.

\section{Estimación e interpretación del parámetro b}

El modelo de Rasch analiza también la validez de constructo con base en la jerarquía de los ítems; se calcula y muestra en el mapa de ítems mediante las estimaciones de dificultad de los mismos. Las unidades logit se interpretan como: (1) frecuencia de procrastinación: cuanto mayor nivel en la escala hay mayor frecuencia de procrastinación del comportamiento que describe el ítem; y (2) razones para procrastinar: a mayor nivel de la escala significa que el motivo descrito en el ítem ejerce mayor influencia para procrastinar una tarea. El Mapa Wright (Figuras 1 y 2) en la columna de la izquierda ubica a las personas por habilidad a lo largo de la variable, con frecuencia las personas tienen una distribución normal. La columna de la derecha ubica cada ítem por su dificultad a lo largo de la variable del más fácil en la parte inferior, al más difícil en la parte superior.

En la Figura 1 (frecuencia de la procrastinación) se observa que el ítem 10 es el de mayor dificultad (discrimina los niveles más elevados de procrastinación) y los ítems 3 y 9 los de menor dificultad (discrimina los niveles más 
Tabla 4. Estimaciones del infit y outfit del segundo atributo (razones para procrastinar) ítems 19 a 44

\begin{tabular}{|c|c|c|c|c|c|}
\hline Pregunta & Puntaje total & Medida & Model S.E. & $\begin{array}{l}\text { INFIT } \\
\text { MNSQ }\end{array}$ & $\begin{array}{c}\text { OUTFIT } \\
\text { MNSQ }\end{array}$ \\
\hline 14 & 783 & .78 & .06 & .99 & .88 \\
\hline 18 & 805 & .71 & .06 & 1.21 & 1.41 \\
\hline 12 & 861 & .54 & .05 & 1.18 & 1.21 \\
\hline 20 & 886 & .47 & .05 & .95 & .86 \\
\hline 7 & 914 & .4 & .05 & 1.07 & 1.06 \\
\hline 15 & 914 & .4 & .05 & .95 & .87 \\
\hline 26 & 919 & .39 & .05 & 1.07 & 1.06 \\
\hline 3 & 961 & .28 & .05 & .91 & .89 \\
\hline 11 & 983 & .23 & .05 & .78 & .79 \\
\hline 22 & 1007 & .17 & .05 & 1.2 & 1.08 \\
\hline 19 & 1021 & .13 & .05 & 1.04 & 1.03 \\
\hline 9 & 1099 & -.04 & .05 & .94 & .89 \\
\hline 17 & 1110 & -.07 & .05 & 1.03 & .97 \\
\hline 8 & 1120 & -.09 & .05 & .8 & .8 \\
\hline 23 & 1123 & -.1 & .05 & .87 & .87 \\
\hline 25 & 1123 & -.1 & .05 & 1.02 & 1.02 \\
\hline 5 & 1148 & -.15 & .05 & 1.03 & 1.03 \\
\hline 16 & 1160 & -.18 & .05 & .93 & 1.04 \\
\hline 10 & 1186 & -.23 & .05 & .83 & .83 \\
\hline 13 & 1189 & -.24 & .05 & .821 & .89 \\
\hline 1 & 1197 & -.25 & .05 & 1.07 & 1.09 \\
\hline 24 & 1261 & -.39 & .05 & 1.14 & 1.11 \\
\hline 4 & 1328 & -.53 & .05 & 1.39 & 1.54 \\
\hline 21 & 1329 & -.53 & .05 & 1.15 & 1.09 \\
\hline 6 & 1437 & -.75 & .05 & 1.19 & 1.15 \\
\hline 2 & 1487 & -.86 & .05 & .93 & 1.02 \\
\hline Media & 1090.4 & 0 & .05 & 1.02 & 1.02 \\
\hline Dt. & 182.3 & .42 & 0 & .14 & .17 \\
\hline
\end{tabular}

Nota: De acuerdo al modelo, para cada una de los ítems se consideran ajustados al modelo los valores MNSQ (infit y outfit) comprendidos entre .5 y 1.5

bajos de procrastinación). En la parte superior de la Figura 1 encontramos que hay un grupo de individuos que no tienen ítems que correspondan a su nivel de habilidad, lo que puede indicar probablemente la necesidad de construir algunos ítems que midan mayor nivel de procrastinación.

En la Figura 2 (razones para procrastinar) se observa en la parte superior que los ítem 32 y 36 (razones de miedo al éxito y toma de riesgos) no tienen sujetos en el cuadrante frente a ellos. En la parte inferior puede observarse que debajo del ítem 20 (correspondiente a dificultades para tomar decisiones) hay personas pero no ítems que les correspondan a su nivel de habilidad. Por lo tanto, basados en estos resultados, la prueba podría necesitar ítems adicionales que incluyan más razones para procrastinar y menos ítems relacionados con razones de miedo al éxito y toma de riesgos.

\section{Funcionamiento de la escala}

Recordemos que las categorías de la prueba son: (1) nunca, (2) pocas veces, (3) algunas veces, (4) habitualmente y (5) siempre. De estas cinco categorías, cuatro $(1,3,4$ y 5) se diferencian a lo largo de las mediciones del atributo. En cambio que la categoría 2 se encuentra dentro del mismo nivel del atributo que la categoría 1. Por lo tanto, las categorías 1 y 2 (nunca y pocas veces) tienden a fundirse en una sola. 
Figura 1. Mapa Wright de personas e ítems para la frecuencia de la procrastinación

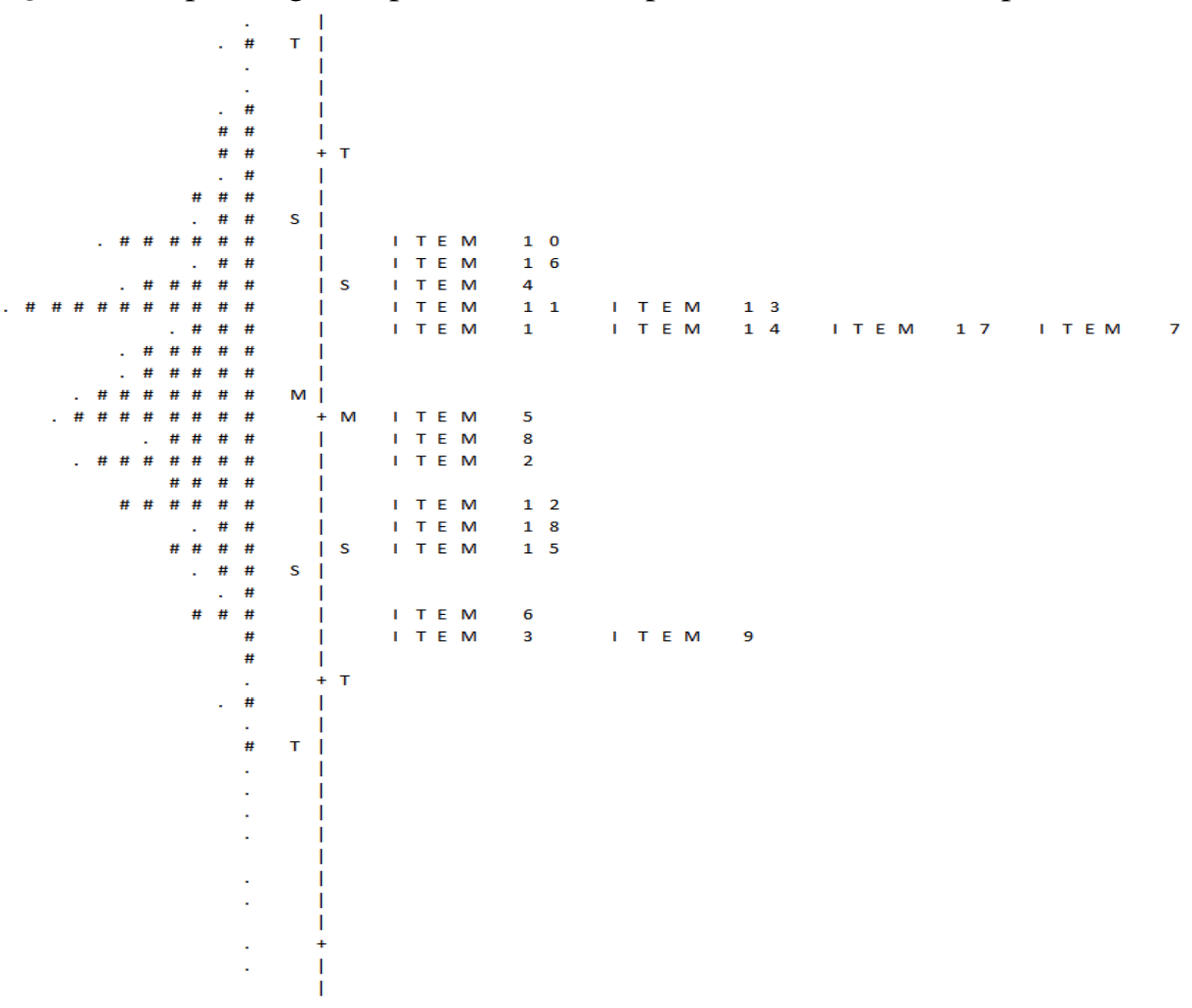

Figura 2. Mapa Wright de personas e ítems para las razones para procrastinar

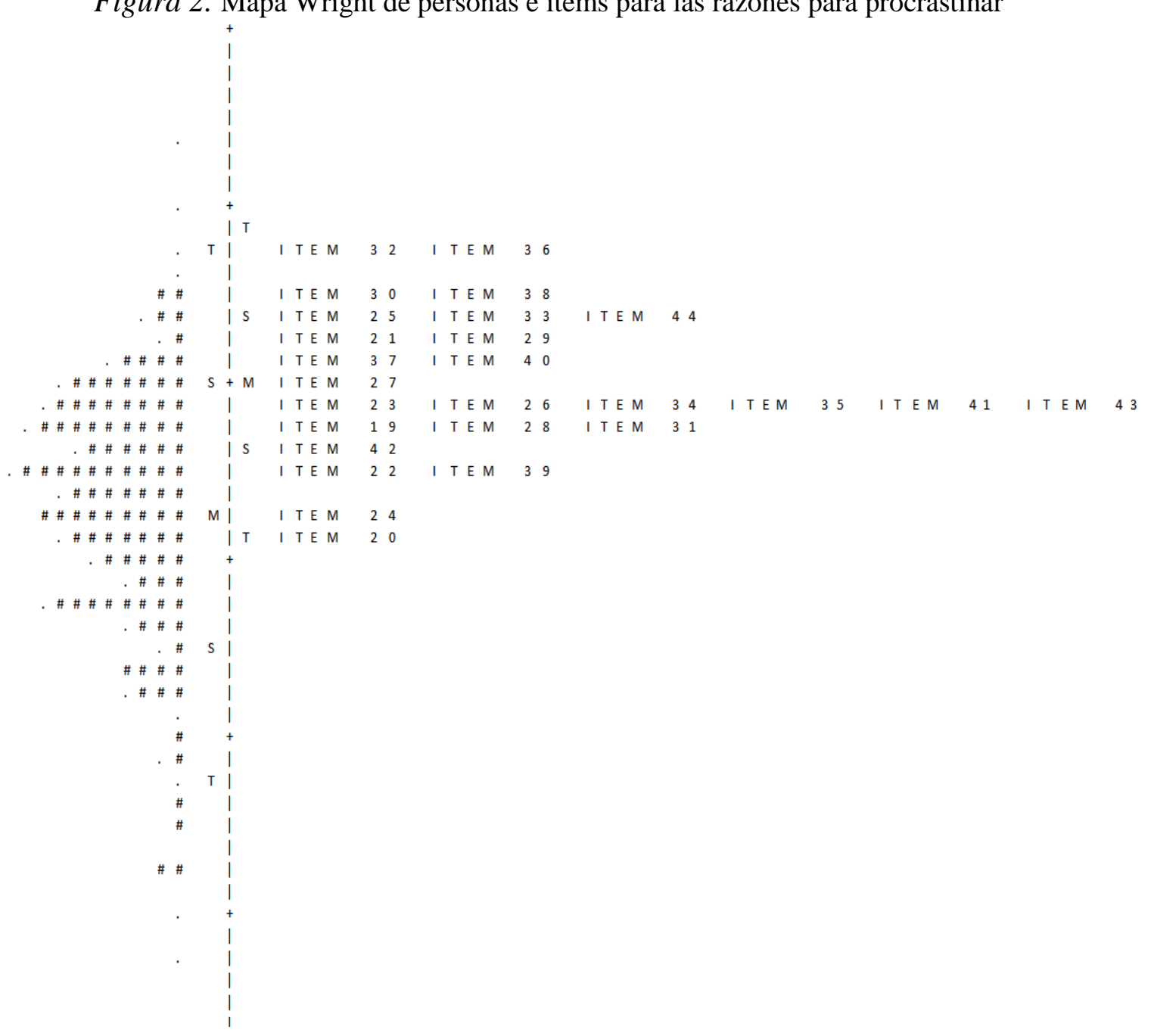




\section{Análisis factorial exploratorio sobre las razones para procrastinar}

La segunda parte de la prueba PASS (ítems 19 a 44) fue construida por Solomon y Rothblum (1984) a partir de un listado general de motivos para procrastinar y ejemplifica teóricamente 14 posibles razones que subyacen a las demoras.
Redactaron dos ítems por cada una de las 14 razones, generando un total de 28 preguntas. Para la presente investigación, se procedió a realizar un "Análisis Factorial Exploratorio (AFE)" con el objetivo de establecer la estructura de las razones para procrastinar de la versión en español del instrumento para estudiantes colombianos.

Tabla 5. Cargas factoriales de cada uno de los ítems sobre las razones para procrastinar

\begin{tabular}{|c|c|c|c|c|c|}
\hline \multirow{2}{*}{ Ítems } & \multicolumn{5}{|c|}{ Componente } \\
\hline & 1 & 2 & 3 & 4 & 5 \\
\hline Te gustó el reto de esperar hasta la fecha de entrega & .742 & .035 & .108 & .010 & .140 \\
\hline Tenías ganas de sentir la emoción de hacer esta tarea en el último momento & .692 & .061 & -.011 & .101 & .295 \\
\hline Estabas preocupado de que si lo hacías bien, tus compañeros te rechazaran & .663 & .012 & .243 & .035 & .374 \\
\hline No te gustó que te pusieran plazos (fechas límites) & .619 & .321 & .297 & .146 & -.057 \\
\hline No te gustó tener que hacer trabajos "mandados" por otros & .590 & .246 & .156 & .396 & -.042 \\
\hline Te sentías demasiado perezoso para escribir un trabajo de final de curso & .088 & .680 & .301 & -.111 & .212 \\
\hline Tenías muchas otras cosas que hacer & -.076 & .645 & -.252 & .083 & .054 \\
\hline Sabías que tus compañeros tampoco habían empezado el trabajo & .436 & .560 & .121 & .092 & -.110 \\
\hline No tenías bastante energía para empezar la tarea & .046 & .551 & .222 & -.206 & .380 \\
\hline No te gusta nada escribir trabajos extensos & .170 & .548 & .150 & .253 & .276 \\
\hline $\begin{array}{l}\text { Esperaste hasta que un compañero hizo el suyo para que te pudiera } \\
\text { aconsejar }\end{array}$ & .332 & .516 & -.030 & .266 & .251 \\
\hline Pensabas que cuesta demasiado tiempo escribir un trabajo de final de curso & .159 & .487 & .393 & .161 & .147 \\
\hline Te sentías desbordado por la tarea & .138 & .484 & .192 & .311 & .204 \\
\hline Tus amigos te presionaban para hacer otras cosas & .378 & .326 & .319 & -.055 & .227 \\
\hline Esperaste a ver si el profesor te ofrecía más información sobre el trabajo & .199 & .306 & .494 & .237 & .090 \\
\hline Te pusiste metas muy altas y te preocupaba no poder alcanzarlas & .115 & .035 & .748 & .197 & .162 \\
\hline $\begin{array}{l}\text { Estabas preocupado de que si recibías una buena nota la gente } \\
\text { esperaría mucho de ti en el futuro }\end{array}$ & .401 & .021 & .644 & .090 & .169 \\
\hline Estabas preocupado de no alcanzar tus propias expectativas & -.012 & .146 & .537 & .377 & .324 \\
\hline Estabas preocupado de que al profesor no le gustara tu trabajo & .227 & -.049 & .148 & .754 & .180 \\
\hline Estabas preocupado de recibir una mala calificación & .066 & .075 & .419 & .711 & .093 \\
\hline Pensabas que no sabías lo suficiente para escribir el trabajo & .262 & .238 & .083 & .482 & .424 \\
\hline No tenías confianza en ti mismo para hacer un buen trabajo & .297 & .123 & .171 & .123 & .663 \\
\hline Tenías problemas en pedir información a otros & .290 & .151 & .198 & .229 & .567 \\
\hline No podías elegir entre todos los posibles temas & .027 & .257 & .181 & .169 & .558 \\
\hline $\begin{array}{l}\text { Necesitabas pedir información al profesor pero no te sentías } \\
\text { cómodo acercándote a el/ella }\end{array}$ & .128 & .326 & .069 & .340 & .419 \\
\hline Tenías dificultades en saber qué incluir y qué no incluir en tu trabajo & -.264 & .341 & .099 & .471 & .317 \\
\hline
\end{tabular}

Nota: Método de extracción de análisis de componentes principales y método de rotación normalización Varimax. En negrita se señala la asignación de cada ítem a uno de los cinco factores 
La idoneidad de este análisis se ha contrastado a partir de diferentes medidas. El test de esfericidad de Bartlett arrojó un chi-cuadrado $(4417 ; p<.001)$ y la medida de Kaiser-MeyerOlkin, de adecuación del muestreo, alcanzó un valor de .913, que puede calificarse de excelente de cara a la aplicación del AFE. Ambos índices corroboran la idoneidad de la matriz de correlaciones entre ítems para llevar a cabo el análisis.

Los resultados indican una adecuada comunalidad de todos los ítems de la prueba, por encima de .30. Por lo tanto, inicialmente ningún ítem fue eliminado por su escasa comunalidad.

El análisis se realizó mediante Componentes Principales, dado que el objetivo de esta técnica es explicar la mayor cantidad de la varianza de las variables originales a través del menos número de factores o componentes. Se realizó una rotación factorial por Varimax.

Para la extracción del número de factores se consideraron criterios teóricos y empíricos. Se consideraron el "scree test" (o gráfico de sedimentación) y que la proporción de la varianza explicada por el factor estuviera por encima del $5 \%$, junto con la interpretabilidad de las estructuras resultantes (Gorsuch, 1983). De acuerdo con las consideraciones anteriormente explicadas, se conservaron los cinco primeros factores de la prueba que explican en total un $54 \%$ de la varianza. Para la asignación de cada una de los ítems a los componentes o factores se tuvo en cuenta la saturación de cada ítem en cada uno de los cinco factores. Los criterios de asignación fueron una saturación igual o superior a .3 al factor y la coherencia teórica del ítem con el contenido del factor (teniendo en cuenta los demás ítems asignados al factor). Las saturaciones factoriales de cada reactivo a cada uno de los cinco factores y su asignación final pueden verse en la tabla 5. Por otra parte, cuando algún ítem presentaba saturaciones factoriales por encima de .3 para más de un factor (por ejemplo, ver en la tabla 5 reactivos 20, 21, 23 o 44), la decisión de su asignación final se tomó teniendo en cuenta: (1) que la saturación del ítem fuera superior al factor asignado que a otros factores; (2) a su contenido, es decir, a su coherencia con los demás ítems asignados previamente al factor y (3) a que el ítem no disminuyera sino que aportara a la confiabilidad de la subescala conformada.

Una vez que se conformaron las subescalas, siguiendo el procedimiento anteriormente señalado, se obtuvo el Alfa de Cronbach para cada uno de los factores resultantes. Los valores de confiabilidad de todas las subescalas fueron superiores a .7; siendo el valor más elevado para el factor 2 (.82) y el más bajo para el factor 3 (.71). Los resultados de la matriz de componentes rotados y los coeficientes de confiabilidad para cada factor pueden verse en la Tabla 6.

Tabla 6. Varianza total explicada por la prueba, por

los factores rotados y resultados en el Alfa de Cronbach para cada uno de los factores

\begin{tabular}{ccccc}
\hline Componente & $\begin{array}{c}\text { Número } \\
\text { de ítems }\end{array}$ & $\begin{array}{c}\text { \% de la } \\
\text { varianza }\end{array}$ & $\begin{array}{c}\% \\
\text { acumulado }\end{array}$ & $\begin{array}{c}\text { Alfa de } \\
\text { Cronbach }\end{array}$ \\
\hline 1 & 5 & 12.8 & 12.8 & .81 \\
2 & 10 & 12.7 & 25.5 & .82 \\
3 & 3 & 9.7 & 35.3 & .71 \\
4 & 2 & 9.6 & 45 & .72 \\
5 & 6 & 9.4 & 54 & .76 \\
\hline
\end{tabular}

Nota: Sumas de las saturaciones al cuadrado de la rotación

Se procedió a asignar un nombre a los cinco factores resultantes de acuerdo al sentido del contenido de los ítems componentes de cada uno de ellos (ver resultados en la Tabla 7).

La primera subescala, "búsqueda de excitación" aglutina razones de búsqueda de activación a través de las demoras y la rebelión, que en la literatura se define como procrastinación por excitación. La segunda subescala, "falta de energía y autocontrol" abarca razones relacionadas con la falta de energía y autocontrol emocional/del entorno (por ejemplo, en cuanto al manejo del tiempo, la dependencia social y la baja tolerancia a la frustración) y, adicionalmente, de un componente de aversión a la tarea. La tercera subescala "perfeccionismo" incluye básicamente el autoperfeccionismo como la característica distintiva; incluso en la pregunta correspondiente a "miedo al éxito": Estabas preocupado de que si recibías una buena nota la gente esperaría mucho de ti en el futuro. Esta pregunta podría ser interpretada como perfeccionismo por su impacto social, para impresionar a otros. La cuarta subescala "ansiedad a la evaluación" claramente 
hace referencia a lo que en la literatura se reporta como el miedo al fracaso/fallo en la tarea. La quinta subescala "poca asertividad y confianza" involucra la percepción de carencia de habilidades para tomar decisiones o habilidades sociales para buscar la información para llevar a cabo una tarea con éxito.

Tabla 7. Ítems, razones para procrastinar y subescala a la que pertenecen

\begin{tabular}{|c|c|c|}
\hline $\begin{array}{c}\text { Categoría de razones } \\
\text { para procrastinar }\end{array}$ & Ítems & Subescala \\
\hline $\begin{array}{l}\text { Rebelión contra el } \\
\text { control }\end{array}$ & 25 y 38 & \multirow{3}{*}{$\begin{array}{l}\text { Búsqueda de } \\
\text { excitación }\end{array}$} \\
\hline Toma de riesgos & 30 y 36 & \\
\hline Miedo al éxito & 32 & \\
\hline $\begin{array}{l}\text { Tendencia a sentirse } \\
\text { desbordado y pobre } \\
\text { manejo del tiempo }\end{array}$ & 22 y 28 & \multirow{6}{*}{$\begin{array}{l}\text { Falta de energía } \\
\text { y autocontrol }\end{array}$} \\
\hline Influencia de pares & 21 y 44 & \\
\hline Aversión a la tarea y & & \\
\hline $\begin{array}{l}\text { baja tolerancia a la } \\
\text { frustración }\end{array}$ & 27 y 35 & \\
\hline Pereza & 34 y 43 & \\
\hline $\begin{array}{l}\text { Dependencia y } \\
\text { búsqueda de ayuda }\end{array}$ & 37 y 41 & \\
\hline Perfeccionismo & 39 y 42 & \multirow{2}{*}{ Perfeccionismo } \\
\hline Miedo al éxito & 40 & \\
\hline $\begin{array}{l}\text { Ansiedad a la } \\
\text { evaluación }\end{array}$ & 19 y 24 & $\begin{array}{c}\text { Ansiedad a la } \\
\text { evaluación }\end{array}$ \\
\hline $\begin{array}{l}\text { Dificultad para tomar } \\
\text { decisiones }\end{array}$ & 20 y 31 & \multirow{3}{*}{$\begin{array}{l}\text { Poca asertividad } \\
\text { y confianza }\end{array}$} \\
\hline Baja asertividad & 23 y 29 & \\
\hline Poca autoconfianza & 26 y 33 & \\
\hline
\end{tabular}

Evidencia de la validez discriminante y predictiva del PASS con otras medidas

Finalizado el periodo académico se obtuvieron las calificaciones ponderadas de cada participante. El sistema de calificaciones colombiano se genera entre un rango de 1 a 5 , donde 3 es el punto de corte para la aprobación de las asignaturas. Se procedió a transformar las calificaciones brutas a puntuaciones típicas estandarizadas para cada una de las titulaciones, obteniendo la calificación estandarizada de acuerdo a los resultados del grupo normativo, en este caso, por carrera.
Como evidencia de validez discriminante $\mathrm{y}$ predictiva entre el PASS y otras medidas, se establecieron las correlaciones entre la frecuencia de procrastinación (primer atributo del PASS), el rendimiento académico, la puntuación general obtenida en el TMB, y con cada una de las cuatro subescalas del TMB (ver Tabla 8).

Tabla 8. Correlaciones entre la frecuencia de procrastinación con las calificaciones y con los resultados en la prueba de gestión del tiempo (TMB)

\begin{tabular}{lc}
\hline \multicolumn{1}{c}{ Pruebas } & Frecuencia PASS \\
\hline TMB total & $-.461^{* *}$ \\
Percepción & $-.371^{* *}$ \\
Herramientas & $-.299^{* *}$ \\
Preferencias & $-.315^{* *}$ \\
Fijar metas & $-.357^{* *}$ \\
Calificaciones & $-.156^{* *}$ \\
\hline : $(*)$ La correlación es significativa al nivel 0.05 \\
$(* *)$ La correlación es significativa al nivel 0.01
\end{tabular}

Como evidencia de validez predictiva, se obtuvo una correlación negativa baja pero significativa al 0.01 entre el rendimiento académico y los resultados en la frecuencia de procrastinación del PASS. Como era de esperarse, a mayor procrastinación menor rendimiento académico

Como evidencia de validez discriminante, se obtuvieron correlaciones inversas significativas al 0,01 con valores entre bajos y moderados, en todas las asociaciones entre el PASS y TMB. Como era de esperarse, a mayor procrastinación menor es la habilidad en la gestión del tiempo de los estudiantes.

\section{Discusión}

El presente estudio tuvo como objeto el análisis de las características psicométricas de la prueba PASS traducida y adaptada en Colombia. Obtuvieron sus principales indicadores psicométricos de confiabilidad y validez, habida cuenta de la falta de constancia hasta la actualidad de ese tipo de estudios en lengua española. 
Se encontraron valores ajustados al modelo Rasch, lo que indica evidencia de validez de constructo de la prueba y un buen funcionamiento de cada uno de los ítems. No obstante, la primera parte de la prueba (frecuencia) podría beneficiarse de la incorporación de ítems que midan mayor nivel de procrastinación y la segunda parte podría mejorar si se incorporan más razones para procrastinar y menos razones de miedo al éxito y toma de riesgos.

Dado que las categorías de respuesta 1 y 2 (nunca y pocas veces) tienden a fundirse en una sola, la prueba podría reducirse a una escala Likert de cuatro categorías.

Por otro lado, los resultados del AFE indican la existencia de cinco factores frente a los dos originalmente encontrados por Solomon $y$ Rothblum (1984). Queda abierta la pregunta sobre la naturaleza de las diferencias entre los resultados en el alumnado colombiano y el anglosajón, respecto a que impliquen un comportamiento diferente del constructo a nivel transcultural o generacional, al menos en relación con las razones que llevan a los individuos a procrastinar.

En cuanto a los resultados que apoyan la validez de criterio discriminante del PASS, los análisis muestran una asociación inversa entre la frecuencia de procrastinación y los resultados en la prueba de gestión del tiempo. A mayor habilidad en gestión del tiempo menor frecuencia de procrastinación. Igualmente, como evidencia de validez predictiva, se encontró una relación inversa entre la procrastinación y el rendimiento académico.

Estos resultados son coherentes con estudios previos que señalan que los procrastinadores tienden a reportar menor uso de estrategias de manejo del tiempo que los no procrastinadores (Lay \& Schouwenburg,1993). Aunque en el presente estudio no se encontró una particular asociación inversa con alguna de las subescalas del TMB, que resultaron relativamente similares para todas las subescalas y significativas al 0.01.

Dado que, según los resultados empíricos la procrastinación y las bajas habilidades de gestión del tiempo van de la mano, la relación entre estas variables podría configurarse en la base de una incapacidad general para poner en marcha las actividades, generando patrones de procrastinación que, en última instancia, conducen a un bajo rendimiento académico.

Por otra parte, los resultados apoyan la afirmación de Moore (2008) quien concluye que la procrastinación reportada mediante cuestionarios puede ser un fuerte predictor de la ejecución académica, en este caso en los primeros semestres. Este resultado abre la perspectiva del empleo de la versión castellana del PASS para la detección temprana de problemas de procrastinación en el contexto de la ejecución de programas preventivos de deserción universitaria en los primeros semestres.

En cuanto a sus limitaciones, cabe destacar la restricción de la muestra, ya que corresponde a un solo país. Sería interesante investigar si los resultados obtenidos con la actual adaptación de la prueba se mantendrían en otros países hispanohablantes. Para futuros estudios también sería recomendable identificar variables moderadoras asociadas a la procrastinación y su efecto sobre el rendimiento académico. Además, se podría utilizar para describir las relaciones estructurales que pueden darse entre diversos factores y estar en condiciones de caracterizar "perfiles de riego" de estudiantes procrastinadores. La identificación temprana de estudiantes procrastinadores conlleva a la necesidad de plantear programas o intervenciones preventivas que permitan reducir la frecuencia de la procrastinación en el alumnado y comprobar la eficacia de esa disminución y la mejora del rendimiento académico.

\section{Referencias}

Bond, T., \& Fox, C. (2012). Applying the Rasch Model: Fundamental measurement in the human sciences. New York: Routledge.

Britton, B., \& Tesser, A. (1991). Effects of timemanagement practices on college grades. Journal of Educational Psychology, 83(3), 405-410. doi: 10.1037/0022-0663.83.3.405

Bruinsma, M., \& Jansen, E. (2009). When will I succeed in my first-year diploma?: Survival analysis in Dutch higher education. Higher Education Research and Development, 28(1), 99-116. 
Burka, J. B., \& Yuen, L. M. (2008). Procrastination: Why you do it, what to do about it now (2nd Ed.). Cambridge, MA: Da Capo Life- long Books.

Dufey, M., \& Fernández, A. (2012). Validity and reliability of the Positive Affect and Negative Affect Schedule (PANAS) in Chilean college students. Revista Iberoamericana de Diagnóstico y Evaluación - e Avaliação Psicológica, 34(1),157-173.

Dewitt, S., \& Schouwenburg, H. (2002). Procrastination, temptations, and incentives: The struggle between the present and the future in procrastinators and the punctual. European Journal of Personality, 16(6), 469489.

Díaz-Morales, Ferrari, J., Díaz, K., \& Argumedo, D. (2006). Factorial Structure of Three Procrastination Scales with a Spanish Adult Population. European Journal of Psychological Assessment, 22(2), 132-137.

Carvalho, R., \& Novo, R. (2014) Personality dimensions and risk behaviors in adolescence: a study with the Portuguese version of the MMPI-A. Revista Iberoamericana de Diagnóstico y Evaluación - e Avaliação Psicológica, 37(1), 203-222.

Ferrari, J., Mason, C., \& Hammer, C. (2006). Procrastination as a predictor of task perceptions: Examining delayed and nondelayed tasks across varied deadlines. Individual Differences Research, 4(1), 28-36.

Ferrari, J.R., O'Callaghan, J., \& Newbegin, I. (2005). Prevalence of procrastination in the United States, United Kingdom, and Australia: Arousal and avoidance delays among adults. North American Journal of Psychology, 7, 1-6.

García-Ros, R., \& Pérez-González, F. (2012). Spanish Version of the Time Management Behavior Questionnaire for University Students. The Spanish Journal of Psychology, 15(3), 1485-1494.

Gorsuch, R. (1983). Factor analysis. Hillsdale, NJ: Erlbaum.

Gortner, A., \& Zulauf, C. (2000). Factors associated with academic time use and academic performance of College students: A recursive approach. Journal of College Student Development, 41(5), 544-556.

Kitsantas, A., Winsler, A., \& Huie, F. (2008). Self-regulation and ability predictors of academic success during college: A predictive validity study. Journal of Advanced Academics, 20(1), 42-68.

Klassen, R., \& Kuzucu, E. (2009). Academic procrastination and motivation of adolescents in Turkey. An International Journal of Experimental Educational Psychology, 29(1), 69-81.

Lay, C. (1986) At last, my research article on procrastination. Journal of Research in Personality, 20, 474-495.

Lay, C., \& Schouwenburg, H. (1993). Trait procrastination, time management, and academic behavior. Journal of Social Behavior \& Personality, 8(4), 647-662.

Linacre, J. M. (2006). A user's guide to winsteps ministeps: Rasch-Model computer programs. Chicago, IL: Electronic Publication. Recuperado de http://ifile.hkedcity.net/1/001/ 950/public/Secondary/EI0020070012/winstep s.pdf

Linacre, M. (2012) Winsteps Tutorial 4. Recuperado de http://www.winsteps.com/a/ winsteps-tutorial-4.pdf

Macan, Th., Shahani, C., Dipboye, R.L. \& Phillips, A.P. (1990). College students' time management: Correlations with academic performance and stress. Journal of Educational Psychology, 82(4), 760-768.

Macan, Th. (1994). Time management: Test of a process model. Journal of Applied Psychology, 79(3), 381-391.

Messick, S. (1989). Validity. The specification and development of tests of achievement and ability. In R . L. Linn (Ed.), Educational Measurement (3th edition) (pp. 13-103). Washington, DC: American Council on Education.

Moore, R. (2008) Academic procrastination and course performance among developmental education students. Research y Teaching in Developmental Education, 24, (2). Recuperado de https://www.questia.com/lib 
rary/journal/1P3-1550972201/academic-

procrastination-and-course-performanceamong

Nunes, I. (2012) Time Perspective Inventory: A validation study. Revista Iberoamericana de Diagnóstico y Evaluación - e Avaliação Psicológica, 34(1), 117-132.

Pehlivan, A. (2013) The effect of the time management skills of students taking financial accounting course on their course grades and grade point averages. International Journal of Business and Social Science, 4(5), 196-199.

Rice, K., Richardson, C., \& Clark, D. (2012) Perfectionism, procrastination, and psychological distress. Journal of Counseling Psychology, 59(2), 288-302.

Robbins, S., Oh, I., Le, H. \& Button, C. (2009). Intervention effects on college performance and retention as mediated by motivational, emotional, and social control factors: Integrated meta-analytic path analyses. Journal of Applied Psychology, 94(5),11631184.

Rozental, A., \& Carlbring, P. (2014). Understanding and treating procrastination: A review of a common self-regulatory failure. Psychology, 5(13), 1488-1502.

Rozental, A., Forsström, D., Nilsson, S., Rizzo, A., \& Carlbring, P. (2014) Group versus internet-based cognitive-behavioral therapy for procrastination: Study protocol for a randomized controlled trial. Internet Interventions 1, 84-89.

Solomon, L., \& Rothblum, E. (1984). Academic procrastination: Frequency and cognitivebehavioral correlates. Journal of Couseling Psychology, 31(4), 503-509.

Steel, P. (2007) The nature of procrastination: A meta-analytic and theoretical review of quintessential self-regulatory failure. Psychological Bulletin 133(1), 65-94.

Steel, P. (2010). Arousal, avoidant and decisional procrastinators: Do they exist?. Personality and Individual Differences, 48(8), 926-934. doi: 10.1016/j.paid.2010.02.025.

Tuckman, B. W. (1991). The development and concurrent validity of the Procrastination Scale. Educational and Psychological Measurement 51, 473-480.
Tuckman, B. W. (2003). The effect of learning and motivation strategies training on college students' achievement. Journal of College Student Development, 44(3), 430-437.

Wang, Z., \& Englander, F. (2010). A crossdisciplinary perspective on explaining student performance in introductory statistics-what is the relative impact of procrastination?. College Student Journal, 44(2), 458-468.

Wilson, B., \& Nguyen, T. (2012). Belonging to tomorrow: An overview of procrastination. International Journal of Psychological Studies, 14(1), 212-217.

Wolters, Ch. (2003). Understanding procrastination from a self-regulated learning perspective. Journal of Educational Psychology, 95(1), 179-187. DOI: 10.1037/0 022-0663.95.1.179

Zarick, L. M., \& Stonebraker, R. (2009). I'll do it tomorrow the logic of procrastination. College Teaching, 57(4), 211-215. 